\title{
ON RINGS OF SETS II.ZERO-SETS
}

\author{
Dedicated to the memory of Hanna Neumann
}

T. P. SPEED

(Received 2 May 1972)

Communicated by G. B. Preston

\section{Introduction}

In an earlier paper [11」 we discussed the problem of when an $(m, n)$-complete lattice $\mathrm{L}$ is isomorphic to an $(m, n)$-ring of sets. The condition obtained was simply that there should exist sufficiently many prime ideals of a certain kind, and illustrations were given from topology and elsewhere. However, in these illustrations the prime ideals in question were all principal, and it is desirable to find and study examples where this simplification does not occur. Such an example is the lattice $\mathbf{Z}(X)$ of all zero-sets of a topological space $X$; we refer to Gillman and Jerison [5] for the simple proof that $Z(X)$ is a $(2, \sigma)$-ring of subsets of $X$, where we denote aleph-zero by $\sigma$.

Lattices of the form $\mathbf{Z}(X)$ have occurred recently in lattice theory in a number of places, see, for example, Mandelker [10] and Cornish [4]. These writers have used such lattices to provide examples which illuminate a number of results concerning annihilators and Stone lattices. We also note that, following Alexandroff, a construction of the Stone-Čech compactification can be given using ultrafilters on $\mathbf{Z}(X)$; the more recent Hewitt realcompactification can be done similarly, and these topics are discussed in [5]. A relation between these two streams of development will be given below.

In yet another context, Gordon [6], extending some aspects of the work of Lorch [9], introduced the notion of a zero-set space $(X, \mathscr{Z})$. This is a structure abstracted from the system consisting of a set $X$ and the family $\mathscr{Z}$ of zero-sets of the functions in a uniformly closed ring of real-valued functions defined on $X$. Gordon's axioms naturally embody some of the lattice-theoretic properties of $\mathbf{Z}(X)$ for a topological space $X$, but as we shall see below, they are more general.

We can now explain the contents of this paper. After listing our notation and terminology, we give some lattice-theoretic results which are necessary for subsequent analysis, but not without interest separately. We then give some 
constructions, similar to Urysohn's, of certain functions separating disjoint sets. They are more delicate than the usual since the family of sets used is closed under (finite unions and) countable intersections only, and hence the notion of closure is not available. Also these results enable us to give alternative proofs of some results of Gordon [6], thus avoiding the use of proximity spaces and the consequent application of Čech's difficult version of Urysohn's lemma, valid for uniformizable proximity spaces. In $\$ \S 4,5$ we turn to the main task which is find properties of $\mathbf{Z}(X)$ in addition to those which follow from its being a $(2, \sigma)$-ring of sets. Our results include algberaic characterisations of $\mathbf{Z}(X)$ for $X$ a compact, respectively arbitrary, topological space.

To conclude this introduction we gratefully thank Drs. J. W. Baker and C. J. Knight for listening to, and helpfully commenting upon, early versions of the material presented below. Also the referee is to be thanked for pointing out an incorrect result stated in the first version, and for remarks leading to some shortening of proofs.

\section{Notation and terminology}

(1.1) Lattice theory. Most of the concepts from lattice theory we need are defined somewhere in Birkhoff [1], while the more special ones relating to rings of sets and special prime ideals are discussed in [11]. All our lattices will be assumed to possess a zero (least element) 0 and unit (greatest element) 1 , and all sublattices will be assumed to contain the same zero and unit. The join and meet operations are denoted $\vee$ and $\wedge$ respectively, and thus a lattice can be considered as an abstract algebra $\mathbf{L}=(L ; \bigvee, \wedge, 0,1)$ with carrier $L$; we use the partial order on $L$ without comment. Typical elements of $L$ will be denoted $a, b, c, d, \cdots$; typical prime or minimal prime ideals will be denoted $w, x, y, \cdots$. We will abbreviate the term $(2, \sigma)$-prime (see [11]) to $\sigma$-prime, in accordance with usual practice. A lattice is said to have enough ideals of a specified type if distinct elements of the lattice can be separated by ideals of that type. The lattice $\mathbf{L}$ is said to be a $(2, \sigma)$-regular sublattice of the lattice $\mathbf{L}^{\prime}$ if $\mathbf{L}$ is a sublattice of $\mathbf{L}^{\prime}$ such that countable meets of elements in $\mathbf{L}^{\prime}$ which exist in $\mathbf{L}^{\prime}$ or $\mathbf{L}$ exist in both and coincide.

(1.2) Topology. Our general reference in this sphere is Bourbaki [2], while the reference for the less common concepts used below, such as zeroset, $z$-filter, realcompactification etc. is Gillman and Jerison [5]. We will reserve $W, X, Y$ for topological spaces; generic elements will be denoted by the corresponding lower case letter; typical subsets will be written $A, B, C, \cdots$; typical open sets $G, \cdots$; typical closed sets $F, \cdots$.

(1.3) General. For subsets $A, B$ of a set $X$ we write $A \cup B, A \cap B$ for set union and intersection respectively, and $\mathbf{C} A$ for the complement of $A$ in $X$. The empty set is denoted $\phi$. If $f: X \rightarrow Y$ is a map, we write $f A$ for the direct image of 
$A \subseteq X$ and $f^{-1} B$ for the inverse image of $B \subseteq Y$; parentheses will only be included where necessary. The unit interval $\{t \in \mathbb{R}: 0 \leqq t \leqq 1\}$ is denoted $[0,1]$.

\section{Some lattice-theoretic results}

Our first definition is based on the work of Cornish [4]; see also Kerstan [7] $\S 6$, Definition 2 for a closely related definition.

Definition 2.1. A lattice $\mathbf{L}$ is normal if for any pair $a, b \in L$ with $a \wedge b=0$, there exists $c, d \in L$ such that $a \wedge c=b \wedge d=0$ and $c \vee d=1$.

It is not hard to see that a Hausdorff space $X$ is normal if, and only if, the lattice $F(X)$ of all closed subsets of $X$ is a normal lattice. Further it has been known for some time that the lattice $\mathbf{Z}(X)$ of all zero-sets of a topological space $X$ is a normal lattice.

A number of equivalent formulations of 2.1 in the case $\mathbf{L}$ a distributive lattice are given in [4], and although we need none of these, we note the following: a distributive lattice $\mathbf{L}$ is normal if, and only if, every prime ideal contains a unique minimal prime ideal. This last result is known for $\mathbf{Z}(X)$ in the form: a prime $z$-filter is contained in a unique $z$-ultrafilter, ([5] 2.13). We also refer to [4] for many consequences of normality. For later use we note that any Boolean lattice is normal.

Another topologically inspired concept we need is that of a $G_{\delta}$-element of a lattice $\mathbf{L}$, and again we note that a similar idea occurs in [7].

DEFINITION 2.2. An element $a \in L$ is a $G_{\delta}$ in the lattice $\mathbf{L}$ if there exists a sequence $\left\{a_{n}: n \geqq 1\right\}$ of (not necessarily distinct) elements of $L$ with the following properties:

( $\alpha) \quad a \wedge a_{n}=0$ for all $n$;

( $\beta)$ if for $b \in L$ we have $b \wedge a_{n}=0$ for all $n$, then $b \leqq a$.

Our final definition in this section is the following abstraction of the analogous topological property.

Definition 2.3. A lattice $\mathbf{L}$ is perfectly normal if $(\alpha) \mathbf{L}$ is normal; and $(\beta)$ every $a \in L$ is a $G_{\delta}$.

Clearly a Hausdorff space $X$ is perfectly normal if, and only if, the lattice $F(X)$ is perfectly normal. Also it is easy to prove $([6] 2.3)$ that for any topological space $X$, the lattice $\mathbf{Z}(X)$ is perfectly normal.

We turn to some algebraic consequences of the definitions.

LEMMA 2.4. A lattice $\mathbf{L}$ in which every $a \in L$ is $a G_{\delta}$ is disjunctive.

ProOF: Take $a \$ b$ in $L$. By $2.2(\beta)$ there must exist an $n$ such that $a \wedge b_{n} \neq 0$ while by $2.2(\alpha) b \wedge b_{n}=0$. This proves the result. 
A deeper result which we use frequently below requires the characteristic property of a minimal prime ideal, Kist [8] viz: a prime ideal $x$ of a distributive lattice $\mathbf{L}$ is minimal if, and only if, for any $a \in x$ there exists $b \notin x$ such that $a \wedge b=0$.

LEMMA 2.5. Let y be a $\sigma$-prime ideal in a $(2, \sigma)$-complete perfectly normal distributive lattice $\mathbf{L}$. Then $y$ is a minimal prime ideal.

ProOF: Let $a \in y$; we must find $b \notin y$ such that $a \wedge b=0$. Since $a$ is a $G_{\delta}$ there exists a sequence $\left\{a_{n}: n \geqq 1\right\}$ with properties $2.2(\alpha),(\beta)$. Thus $a \wedge a_{n}=0$, and so normality of $L$ implies the existence of two sequences $\left\{c_{n}\right\},\left\{d_{n}\right\}$ with: $a \wedge c_{n}=0=a_{n} \wedge d_{n}$ and $c_{n} \vee d_{n}=1$ for all $n$. If, for some $n, c_{n} \notin y$, then we are through. Suppose now that $c_{n} \in y$ for all $n$; then $d_{n} \notin y$ for all $n$, and by the $\sigma$-prime property of $y, d=\wedge_{n} d_{n} \notin y$. But for all $n, a_{n} \wedge d \leqq a_{n} \wedge d_{n}=0$ and so by $2.2(\beta)$ $d \leqq a$ which contradicts $a \in y, d \notin y$.

Hence $a \wedge c_{n}=0$ for some $c_{n} \notin y$ and $y$ is minimal.

\section{Constructions similar to Urysohn's}

In this section we will be working with a $(2, \sigma)$-ring of subsets of a set $X$ satisfying various conditions, and a careful analysis will enable us to extend the construction of a continuous function separating disjoint closed sets to this situation. We conclude by giving an alternative, direct, proof of a result of Gordon.

THEOREM 3.1. Let $\mathbf{H}$ be a $(2, \sigma)$-ring of subsets of a set $X$. Then the following are equivalent:

1) $\mathbf{H}$ is a normal lattice.

2) For any $A, B \in H$ with $A \cap B=\phi$ there exists a function $f: X \rightarrow[0,1]$ such that

( $\alpha) f^{-1} F \in H$ for every closed subset $F$ of $[0,1]$;

(B) $A \subseteq f^{-1}\{0\}, B \subseteq f^{-1}\{1\}$.

ProOF: 1) implies 2). We will explain the proof backwards thus motivating the construction. Let $A, B \in H$ with $A \cap B=\phi$ be given. Our aim is to define a system

$$
\mathscr{U}=\{U(t), F(t): 0 \leqq t \leqq 1\} \text { where }
$$

(i) $\mathrm{G} U(t) \in H, F(t) \in H, 0 \leqq t \leqq 1$;

(ii) $A \subseteq U(0), B \subseteq \mathrm{C} U(1)$;

(iii) If $0 \leqq t<t^{\prime} \leqq 1$ then $U(t) \subseteq F(t) \subseteq U\left(t^{\prime}\right)$.

Then we will see that the well-known procedure of defining a map $f: X \rightarrow[0,1]$ by writing, for $x \in X$ :

$$
f(x)=\inf \{t: x \in U(t)\}
$$


gives a function satisfying:

(iv) $f^{-1}[0, t]=F(t), f^{-1}[t, 1]=\mathbf{C} U(t)$.

Having done this we may take an arbitrary closed subset $[0,1] \backslash \bigcup_{n}\left(\alpha_{n}, \beta_{n}\right)$ of $[0,1]$ and find that

$$
\begin{aligned}
f^{-1}[0,1] \backslash \bigcup_{n}\left(\alpha_{n}, \beta_{n}\right) \\
=f^{-1} \bigcap_{n}\left\{\left[0, \alpha_{n}\right] \cup\left[\beta_{n}, 1\right]\right\} \\
=\bigcap_{n}\left\{f^{-1}\left[0, \alpha_{n}\right] \cup f^{-1}\left[\beta_{n}, 1\right]\right\} \\
\quad \in H \text { as required. }
\end{aligned}
$$

Thus our function $f$ so constructed satisfies $(\alpha)$ and $(\beta)$ of (3.1)2) above.

An so we turn to defining the system $\mathscr{U}$. To do this we first define a subsystem $\mathscr{U}_{\Delta}$, where $\Delta$ is the set of binary rationals in $[0,1]$ :

$$
\mathscr{U}_{\Delta}=\{U(\delta), F(\delta): \delta \in \Delta\} \text { where }
$$

(i)' $\mathrm{C} U(\delta) \in H, F(\delta) \in H, \delta \in \Delta$;

(ii)' $A \subseteq U(0), B \subseteq \mathrm{C} U(1)$;

(iii)' If $0 \leqq \delta<\delta^{\prime} \leqq 1$ then $U(\delta) \subseteq F(\delta) \subseteq U\left(\delta^{\prime}\right)$.

Let us suppose for the moment that $\mathscr{U}_{\Delta}$ is defined and satisfies (i)', (ii)' and (iii)'. Then if we write, for $0 \leqq t \leqq 1$ :

$$
U(t)=\bigcup_{\delta>t} U(\delta), F(t)=\bigcap_{\delta>t} F(\delta),
$$

we clearly obtain a system $\mathscr{U}$ satisfying (i) and (ii). We check (iii). Take $t, t^{\prime}$ with $0 \leqq t<t^{\prime} \leqq 1$; there exists $\delta, \delta^{\prime}, \delta^{\prime \prime} \in \Delta$ with $t<\delta<\delta^{\prime}<\delta^{\prime \prime}<t^{\prime}$, and so by (iii)' and $(\dagger)$

$$
U(t) \subseteq U(\delta) \subseteq F(\delta) \subseteq U\left(\delta^{\prime}\right) \subseteq F\left(\delta^{\prime}\right) \subseteq U\left(\delta^{\prime \prime}\right) \subseteq U\left(t^{\prime}\right) .
$$

Clearly $F(\delta) \subseteq F(t) \subseteq F\left(\delta^{\prime}\right)$ follows from (†) and so with the above we obtain

$$
U(t) \subseteq F(\delta) \subseteq F(t) \subseteq F\left(\delta^{\prime}\right) \subseteq U\left(t^{\prime}\right) \text { which implies (iii). }
$$

Thus our problem reduces to constructing $\mathscr{U}_{\Delta}$ satisfying (i) ${ }^{\prime}$, (ii)', (iii)'. This is done inductively, using the representation $\Delta=\left\{k 2^{-m}: k=0,1, \cdots, 2^{m} ; m \geqq 0\right\}$; we define for $m \geqq 0$ :

$(*)^{\prime \prime}$

$$
\mathscr{U}_{m}=\left\{U\left(k 2^{-m}\right), F\left(k 2^{-m}\right): 0 \leqq k \leqq 2^{m}\right\} \text { where }
$$

(i) $^{\prime \prime} \quad \mathrm{C} U\left(k 2^{-m}\right) \in H, F\left(k 2^{-m}\right) \in H, 0 \leqq k \leqq 2^{m}$;

(ii)" $A \subseteq U(0), B \subseteq \mathrm{CU}(1)$;

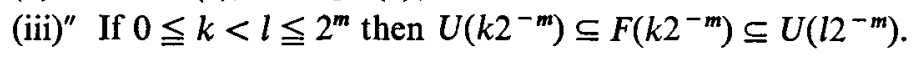

Then we put $\mathscr{U}_{\Delta}=\bigcup_{m \geqq 0} \mathscr{U}_{m}$. 
Case $m=0$. Put $\mathscr{U}_{0}=\{U(0), F(0), U(1), F(1)\}$ where

$$
U(1)=\mathbf{C} B, F(1)=X \text {, and }
$$

$U(0), F(0)$ are chosen so that $\mathrm{C} U(0) \in H, F(0) \in H$, and

$$
A \subseteq U(0) \subseteq F(0) \subseteq \mathrm{C} B .
$$

This can be done: for $A \cap B=\phi$ implies, by the assumed normality of $H$, the existence of $V, F \in H$ with:

$$
A \cap V=\phi, B \cap F=\phi \text { and } V \cup F=X .
$$

But these relations imply $A \subseteq \mathbf{C} V, F \subseteq \mathbf{C B}$ and $\mathbf{C} V \subseteq F$ so that putting $U(0)$ $=\mathrm{C} V$ with $F(0)=F$ satisfies our requirements.

Now suppose that for some $m \geqq 1, \mathscr{U}_{m-1}$ is defined and satisfies (i)", (ii)" and (iii)", and let us consider $\mathscr{U}_{m}$. For even $k$ we define $U\left(k 2^{-m}\right), F\left(k 2^{-m}\right)$ in the obvious way. For odd $k \geqq 1$ we note that (iii)" implies:

$$
U\left((k-1) 2^{-m}\right) \subseteq F\left((k-1) 2^{-m}\right) \subseteq U\left((k+1) 2^{-m}\right) .
$$

The last inclusion can be written $F\left((k-1) 2^{-m}\right) \cap C U\left((k+1) 2^{-m}\right)=\phi$ and so we may proceed as for the case $m=0$ with $F$ replacing $A, C U$ replacing $B$, and find elements $V, F$ of $H$ with

$$
F\left((k-1) 2^{-m}\right) \subseteq \mathrm{C} V \subseteq F \subseteq U\left((k+1) 2^{-m}\right) .
$$

Thus we may put $U\left(k 2^{-m}\right)=\mathrm{CV}$ and $F\left(k 2^{-m}\right)=F$ and satisfy (iii)" thus completing the inductive step.

And so we have constructed $\mathscr{U}_{\Delta}$ and thus $\mathscr{U}$, and it only remains to prove (iv) is valid in order to complete the proof of 1) implies 2). Recall the definition (**) of $f$.

If $f(x) \leqq t$ for some $t \in[0,1]$ then for any $\delta \in \Delta$ with $\delta>t$ we have $x \in U(\delta) \subseteq F(\delta)$ whence $x \in \bigcap_{\delta>t} F(\delta)=F(t)$.

On the other hand, if $x \in F(t)$, then for any $\delta \in \Delta$ with $\delta>t$ we may find $\delta^{\prime} \in \Delta$ with $\delta>\delta^{\prime}>t$ and so $x \in F\left(\delta^{\prime}\right) \subseteq U(\delta)$. Thus $f(x)<\delta$ for each $\delta>t$ whence $f(x) \leqq t$, and we have proved that $f^{-1}[0, t]=F(t)$. The other part of (iv) is proved similarly.

2) implies 1) Let us assume that $A \cap B=\phi$ for $A, B \in H$. By 2) there exists $f: X \rightarrow[0,1]$ such that $A \subseteq f^{-1}\{0\}, B \subseteq f^{-1}\{1\}$ and $f^{-1} F \in H$ for each closed $F \subseteq[0,1]$. If we take $F=\left[0, \frac{1}{2}\right]$ and $\left[\frac{1}{2}, 1\right]$ we obtain $D=f^{-1}\left[0, \frac{1}{2}\right] \in H$, $C=f^{-1}\left[\frac{1}{2}, 1\right] \in H$ such that $A \cap C=\phi, B \cap D=\phi$ and $C \cup D=X$, as required.

REMARK 3.2. If $\mathbf{H}=\mathbf{F}(X)$ is the lattice closed subsets of a topology on $X$ then 3.1 is just Urysohn's lemma. Another special case is when $\mathbf{H}=\mathbf{Z}(X)$ is the lattice of all zero-sets of a completely regular (Hausdorff) space $X$. In this case we have proved (cf. [5] 1.15) that disjoint zero-sets can be separated by a continuous function. 
Our application of 3.1 is in the following result:

THEOREM 3.3. Let $\mathbf{H}$ be a normal $(2, \sigma)$-ring of subsets of a set $X$. Then the following are equivalent for an element $A \in H$ :

1) There exists a sequence $\left\{A_{n}: n \geqq 1\right\}$ of elements of $H$ with the following properties:

(a) $A \cap A_{n}=\phi$ for all $n$;

( $\beta$ ) if for $B \in H$ we have $B \cap A_{n}=\phi$ for all $n$, then $B \subseteq A$.

2) There exists a function $f: X \rightarrow[0,1]$ such that

( $\alpha) f^{-1} F \in H$ for every closed subset $F$ of $[0,1]$;

(B) $A=f^{-1}\{0\}$.

Proof: 1) implies 2). By 3.1 there exists $f_{n}: X \rightarrow[0,1]$ satisfying 2) ( $\alpha$ ) such that $A \subseteq f_{n}^{-1}\{0\}$ and $A_{n} \subseteq f_{n}^{-1}\{1\}$. This uses only 1) ( $\alpha$ ) and works for all $n$. Now consider the element $\bigcap_{n \geqq 1} f_{n}^{-1}\{0\}$ of $H$; clearly $A \subseteq \bigcap_{n \geqq 1} f_{n}^{-1}\{0\}$, and for any $n$,

$$
A_{n} \cap \bigcap_{n \geqq 1} f_{n}^{-1}\{0\} \subseteq f_{n}^{-1}\{1\} \cap \bigcap_{n \geqq 1} f_{n}^{-1}\{0\}=\phi .
$$

Thus by 1) $(\beta) A \supseteq \bigcap_{n \geqq 1} f_{n}^{-1}\{0\}$ and if we define $f: X \rightarrow[0,1]$ by

$$
f=\sum_{n \geqq 1} 2^{-n} f_{n}
$$

it is easy to see $f^{-1}\{0\}=\bigcap_{n \geqq 1} f_{n}^{-1}\{0\}=A$, and Lemma 2.4 of [6] implies that $f$ satisfies 2) ( $\alpha$ ). This completes the proof of the first implication.

2) implies 1). If $A=f^{-1}\{0\}$ for a function $f$ satisfying 2) ( $\beta$ ), then we may define $A_{n}=f^{-1}[1 / n, 1]$ for $n \geqq 1$, With this definition

$$
\mathrm{C} A=\bigcup_{n \geqq 1} A_{n}
$$

and conditions 1) $(\alpha), 1)(\beta)$ are readily checked.

The following corollary can easily be proved using the two previous results.

COROLLARY 3.4. Let $\mathbf{H}$ be a $(2, \sigma)$-ring of subsets of $X$. Then the following are equivalent:

1) $\mathbf{H}$ is a perfectly normal lattice.

2) For every $A \in H$ there exists a function $f: X \rightarrow[0,1]$ such that:

$(\alpha) f^{-1} F \in H$ for every closed subset $F$ of $[0,1]$;

(B) $A=f^{-1}\{0\}$.

In the terminology we are using, a zero-set space is a pair $(X, \mathscr{Z})$ where $X$ is a set and $\mathscr{Z}$ is a perfectly normal $(2, \sigma)$-ring of subsets of $X$ which separates points of $X$. With any such space Gordon associates the set $S(X, \mathscr{Z})$ of all functions $f: X \rightarrow \mathbb{R}$ such that $f^{-1} F \in \mathscr{Z}$ for every closed subset $F$ of $\mathbb{R}$; such functions are called zero- 
set functions. The lemma ([6] 2.4) used in the previous proof shows that $S(X, \mathscr{Z})$ is a uniformly closed ring of functions on $X$; also $S(X, \mathscr{Z})$ separates points of $X$ and contains the constant functions.

We give a new proof of [6] 3.5 viz:

THEOREM 3.5. Let $(X, \mathscr{Z})$ be a zero-set space and $S(X, \mathscr{Z})$ the family of all zero-set functions on $X$. Then

$$
\mathscr{Z}=\{Z(f): f \in S(X, \mathscr{Z})\} .
$$

Proof. By 3.4 every $A \in \mathscr{Z}$ is the zero-set of a suitable function of $S(X, \mathscr{Z})$; if $f \in S(X, \mathscr{Z})$ then $Z(f)=f^{-1}\{0\} \in \mathscr{Z}$ and the proof is complete.

\section{The lattice $Z(X)$ for $X$ compact}

It is well known that a completely regular space $X$ is compact if, and only if, every $z$-ultrafilter is fixed. However, as in the case of the ring $C(X)$, the notion of fixed (resp. free) is not a lattice-theoretic invariant and so we must proceed slightly differently. At this point also, our treatment begins to differ from that in [6] since we only have the lattice $Z(X)$ and not $X$ itself.

The main result of this section is given a proof independently of the discussion in the next section, although it can also be derived from results there. We do this because the simplications which occur when $X$ is compact allow quite different techniques to be used.

THEOREM 4.1. Let $\mathbf{L}$ be a lattice. Then the following are equivalent:

1) $\mathbf{L}$ is isomorphic to the lattice $\mathbf{Z}(X)$ for a compact space $X$.

2) $(\alpha) \mathbf{L}$ is a $(2, \sigma)$-complete lattice;

$(\beta)$ Every minimal prime ideal of $\mathbf{L}$ is $\sigma$-prime;

$(\gamma) \mathbf{L}$ is perfectly normal.

The space $X$ of 1) is NOT unique up to homeomorphism.

Proof. 1) implies 2). We will show that for any compact space $X$ the lattice $\mathbf{Z}(X)$ has properties 2$)(\alpha),(\beta),(\gamma)$; these are obviously lattice invariants and so the implication will be proved. But we have already noted the validity of $(\alpha),(\gamma)$ for $X$ general, and $(\beta)$ follows since every minimal prime ideal of $\mathbf{Z}(X)$ is exactly those elements not belonging to a particular fixed ultrafilter $u_{x}=\{a \in \mathbf{Z}(X): x \in a\}$, where $x \in X$ is unique. Clearly such a minimal prime is $\sigma$-prime, completing the proof.

2) implies 1) Suppose we are given a lattice $\mathbf{L}$ satisfying $(\alpha),(\beta),(\gamma)$ of 2). Let $X$ denote the set of all minimal prime ideals of $\mathbf{L}$, and equip $X$ with the topology whose closed sets are intersections of the sets in $L^{\prime}=\left\{X_{a}: a \in L\right\}$ where for $a \in L, X_{a}=\{x \in X: a \notin x\}$. We will prove that $X$ so defined is a compact 
(Hausdorff) space, and that the family of all zero-sets of $X$ is exactly $\mathbf{L}^{\prime}$, a lattice which will be shown to be isomorphic to $\mathbf{L}$.

We prove this last remark first. Condition 2) $(\gamma)$ together with Lemma 2.5 above implies that $\mathbf{L}$ is disjunctive, and so by a result which is well known (see e.g. [8]) $a \rightarrow X_{a}$ is bijective. It can be readily checked that for $a, b \in L, X_{a} \cup X_{b}$ $=X_{a v b}$, and since the ideals in $X$ are all $\sigma$-prime, we find that for $\left\{a_{n}: n \geqq 1\right\}$ $L, \bigcap_{n \geqq 1} X_{a_{n}}=X_{a}$ where $a=\wedge_{n \geqq 1} a_{n}$. The latter exists, of course, by 2) ( $\alpha$ ).

The proof that $X$, so topologised, is a compact (Hausdorff) space given (in a dual form) in [4] Theorem 7.3 hence we omit it.

And so it remains to prove that $L^{\prime}$ is exactly the family of all zero-sets of $X$. Now $X$ is normal and so it is enough to prove that $L^{\prime}$ is exactly the set of all closed $G_{\delta}$-subsets of $X$. But every $a \in L$ is a $G_{\delta}$ and this is easily seen to imply

$$
\mathrm{C} X_{a}=\bigcup_{n \geqq 1} X_{a_{n}}
$$

proving that $X_{a}$ is a $G_{\delta}$-subset, by definition, closed, of $X$. This proves half of what is required, and to complete the proof we take an arbitrary closed $G_{\delta}$-subset $F$ of $X$. By definition, there is a sequence $M_{n}$ of subsets of $L$, and a subset $B \subseteq L$ such that

$$
\bigcap_{b \in B} X_{b}=F=\bigcap_{n \geqq 1}\left\{\bigcup_{a \in M_{-}} \mathbf{C} X_{a}\right\} .
$$

We concentrate on the right-hand equality first. Since $F$ is compact, for any $n \geqq 1$ there is a finite subset $m_{n} \subseteq M_{n}$ such that

$$
F \subseteq \underset{a \in m_{-}}{\bigcup} \mathbf{C} X_{a}=\mathbf{C} X_{a_{n}},
$$

where $a_{n}=\bigwedge_{a \in m_{n}} a$. Thus we see that

$$
\bigcap_{b \in B} X_{b}=F=\bigcap_{n \geqq 1} \mathbf{G} X_{a_{n}} .
$$

Now each $X_{a_{n}}$ is compact, and so for each $n$ there is a finite subset $B_{n} \subseteq B$ such that

$$
X_{b_{n}}=\bigcap_{b \in B_{n}} X_{b} \subseteq \mathrm{C} X_{a_{n}},
$$

where $b_{n}=\wedge_{b \in B_{n}} b$. Putting these results together gives

$$
F=\bigcap_{n \geqq 1} \mathbf{C} X_{u_{n}} \supseteq \bigcap_{n \geqq 1} X_{b_{n}} \supseteq \bigcap_{b \in B} X_{b}=F,
$$

whence $F=\bigcap_{n \geqq 1} X_{b .}=X_{b}$, where $b=\wedge_{n \geqq 1} b_{n}$, and in this last step we have used the fact that $b \rightarrow X_{b}$ is a $(2, \sigma)$-homomorphism. The proof is now complete.

An interesting byproduct which will be explained in the next section is the following: 
COROLLARY 4.2. Let $Y$ be a pseudocompact topological space. Then there is a compact space $X$ such that $\mathbf{Z}(Y)$ and $Z(X)$ are lattice isomorphic.

Proof. Putting together 5.8(b) and 5.14 of [5] we find that for $Y$ pseudocompact $\mathbf{Z}(Y)$ satisfies (4.1) 2) ( $\beta$ ) and so the result follows.

In particular, the corollary shows that non-homeomorphic pseudocompact spaces can have isomorphic lattices of zero-sets. We will see that this cannot happen when the spaces are both realcompact. Finally, we note that the space $X$ in 4.2 can be taken to be $\beta Y$, or any space $Y \subseteq X \subseteq \beta Y$.

\section{The lattice $Z(X)$ for a general $X$}

In this section we characterise the lattice $\mathbf{Z}(X)$ algebraically, for a general topological space $X$. We begin with a reduction, relying heavily upon results from [5].

Proposition 5.1. For every topological space $X$ there exists a completely regular space $Y$ and a continuous map $\tau$ of $X$ onto $Y$ such that the map:

$$
Z_{Y}(g) \rightarrow Z_{A}(g \circ \tau)
$$

is an isomorphism of $\mathbf{Z}(Y)$ onto $\mathbf{Z}(X)$.

Proof. See [5] 3.9. The details are easy, and omitted. The next stage of our reduction is again similar to the ring case.

Proposition 5.2. For every completely regular space $X$ there exists a realcompact space $\cup X$ and a continuous map $\tau$ of $X$ into $\cup X$ such that the map:

$$
a \rightarrow c l_{v X^{a}}
$$

is an isomorphism of $\mathbf{Z}(X)$ onto $\mathrm{Z}(\mathrm{v} X)$ ).

Proof. See [5] 8.8.

From now on we will suppose, where appropriate, that $X$ is realcompact. As a first attack on our characterisation problem we abstract the lattice-theoretic properties of a zero-set structure.

Definition 5.3. A lattice $\mathbf{L}$ is a $z$-lattice if

$(\alpha) \mathbf{L}$ is $(2, \sigma)$-complete;

$(\beta) \mathbf{L}$ has enough $\sigma$-prime minimal prime ideals;

$(\gamma) \mathbf{L}$ is perfectly normal.

For any topological space $X$ the lattice $\mathbf{Z}(X)$ is a $z$-lattice. To see this we need only check $(\beta)$ as $(\alpha)$ and $(\gamma)$ of 5.3 have already been noted. Now for any $x \in X$ the family $j_{x}=\{a \in \mathbf{Z}(X): x \notin a\}$ is easily seen to be a $\sigma$-prime ideal of $\mathbf{Z}(X)$ and 
there are certainly enough of these ideals to distinguish elements of $\mathbf{Z}(X)$. Thus $(\beta)$ will be satisfied if we show that all the $\sigma$-prime ideals $j_{x}$ are minimal. But this follows from 2.5 above; alternatively a direct proof can be given.

We will see below that although not every $z$-lattice is isomorphic to a lattice $\mathbf{Z}(X)$, such a lattice can be embedded as a sublattice of $\mathbf{Z}(X)$ for a suitable $X$ in a particularly precise manner, which it is convenient to formulate separately. For any $z$-lattice $\mathbf{L}$ (possibly with superscripts) we denote by $X^{\mathrm{L}}$, or just $X$ if no ambiguity is possible, (with the same superscripts) the set of all $\sigma$-prime minimal prime ideals of $\mathbf{L}$; for $a \in L$ we write $X_{a}^{\mathrm{L}}=X_{c}=\left\{x \in X^{\mathrm{L}}: a \notin x\right\}$.

Definition 5.4. A $z$-lattice $\mathbf{L}$ is said to be a $z$-sublattice of the $z$-lattice $\mathbf{L}^{\prime}$, equivalently, $\mathbf{L}^{\prime}$ is a $z$-extension of $\mathbf{L}$, if

$(\alpha) \mathbf{L}$ is a $(2, \sigma)$ regular sublattice of $\mathbf{L}^{\prime}$;

$(\beta)$ the map $x^{\prime} \rightarrow x^{\prime} \cap L$ is a bijection from $X^{\prime}$ onto $X$;

( $\gamma$ ) for any $b \in L^{\prime}, X_{b}^{\prime}=\bigcap\left\{X_{a}^{\prime}: a \in L, a \geqq b\right\}$.

We will see that the property of being a $z$-sublattice is transitive, a fact needed below.

LEMMA 5.5. If $\mathbf{L}$ is a z-sublattice of $\mathbf{L}^{\prime}$, and $\mathbf{L}^{\prime}$ is a $z$-sublattice of $\mathbf{L}^{\prime \prime}$, then $\mathbf{L}$ is a $z$-sublattice of $\mathbf{L}^{\prime \prime}$.

Proof. Clearly $(\alpha)$ and $(\beta)$ are true so we need only prove $(\gamma)$. Let $b \in L^{\prime}$. We will show that

$$
X_{b}=\bigcap\left\{X_{a}: a \in L, a \geqq b\right\}
$$

is true, and then the fact that for any $c \in L^{\prime \prime}$

$$
X_{c}^{\prime \prime}=\bigcap\left\{X_{b}^{\prime \prime}: b \in L^{\prime}, b \geqq c\right\}
$$

will complete the proof. Now suppose that $x^{\prime \prime} \in X^{\prime \prime}$ is such that $a \notin x^{\prime}$ for all $a \in L$ with $a \geqq b$. Then $a \notin x^{\prime \prime} \cap L^{\prime}=x^{\prime}$ say, for all $a \in L$ such that $a \geqq b$, and so $b \notin x^{\prime}$, since $L^{\prime}$ is a $z$-extension of $L$. Thus we have proved $b \notin x^{\prime \prime}$ and the equality $\left(^{*}\right)$ is proved.

The following results is the main step in our characterisation theorem.

THEOREM 5.6. Let $\mathbf{L}$ be a $z$-lattice. Then $X=X^{\mathrm{L}}$ is a realcompact space, and $\mathbf{L}$ is isomorphic to a $z$-sublattice of the $z$-lattice $Z(X)$.

Proof. We give $X$ the topology whose closed subsets are intersections of sets in $L^{\prime}=\left\{X_{a}: a \in L\right\}$. Exactly as in 4.1 above we can prove that $\mathbf{L}$ is isomorphic to $\mathbf{L}^{\prime}$ where $\mathbf{L}^{\prime}$ is the set $L^{\prime}$ under the operations of finite set-union and countable set-intersection; the isomorphism is a $(2, \sigma)$-homomorphism. 
Thus $\mathbf{L}^{\prime}$ is a $z$-lattice, and so the results of $\S 3$ above will apply. We prove that $X$ is completely regular. Take a point $x \in X$ and a closed set $F=\bigcap\left\{X_{a}: a \in \mathbf{M}\right\}$ not containing $x$. Then there is $X_{a} \subseteq F$ with $x \notin X_{a}$; since $a$ in $L$ and hence $X_{a}$ in $L^{\prime}$ is a $G_{\partial}, x \in X_{b}$ with $X_{a} \cap X_{b}=\phi$ for a suitable $b \in L$. By Theorem 3.1 there is a continuous function $f: X \rightarrow[0,1]$ with $f(x)=1$ and $f^{-1}\{0\} \supseteq F$. Now Corollary 3.4 shows that every element of $L^{\prime}$ is a zero-set of $X$ and so $L \subseteq Z(X)$. Before we show that $\mathbf{L}^{\prime}$ is a $z$-sublattice of $\mathbf{Z}(X)$ it will be necessary to prove that $X$ is realcompact. Let $\eta$ be a real $z$-ultrafilter on $X$; then $y=\{a \in Z(X): a \notin \eta\}$ is a $\sigma$-prime minimal prime ideal on $\mathbf{Z}(X)$, and so $y^{\prime}=y \cap L^{\prime}$ is a $\sigma$-prime ideal of $\mathbf{L}^{\prime}$. Lemma 2.5 implies that $y^{\prime}$ is in fact a minimal prime ideal, and so $y^{\prime}=x$ for some unique $x \in X$. Now the intersection of all the zero-sets in $\eta$ is, by the definition of the topology, an intersection of all the zero-sets of the form $X_{a}$ in $\eta$ and this intersection contains $x$; thus $X$ is realcompact by [5] 5.15 .

Having now established that $\mathbf{L}$ is isomorphic to the sublattice $\mathbf{L}^{\prime}$ of the lattice $\mathbf{Z}(X)$ where $X$ is a realcompact space, our proof is completed by proving that $\mathbf{Z}(X)$ is a $z$-extension of $\mathbf{L}^{\prime}$. This is really quite easy once we observe that the $z$-lattice $Z(X)$ has a space $X^{Z(X)}$ of $\sigma$-prime minimal prime ideals which is canonically homeomorphic to $X$ under the map $x \rightarrow j_{x}=\{a \in Z(X): x \notin a\}$. Referring to 5.4 we see that $(\alpha)$ is valid, $(\beta)$ follows from Lemma 2.5 and the preceding remark, and $(\gamma)$ simply expresses the fact that every zero-set in $X$ (more precisely, its homeomorph $\left.X^{2(X)}\right)$ is closed and hence an intersection of the basic closed sets in $L^{\prime}$ (more precisely, their copies inside $X^{Z(X)}$ ). Thus the theorem is proved.

It might have been hoped that in the previous construction, $\mathbf{L}^{\prime}$ actually coincides with $\mathbf{Z}(X)$, but as already observed, this is not generally so. After examining an example which validates this assertion we formulate and prove the maximality property possessed by lattices $Z(X)$, and our main characterisation theorem quickly follows.

EXAmple 5.7. Consider the $z$-lattice $\mathbf{B}=\mathbf{B}[0,1]$ of all Borel subsets of $[0,1]$. Then $\mathbf{B}$ is a $z$-sublattice of the power set $\mathbf{P}=\mathbf{P}[0,1]$.

Proof. To see this we also need to refer to $\mathbf{F}=\mathbf{F}[0,1]$, the $z$-lattice of all closed sets (= zero-sets) of $[0,1]$ with the usual topology. Before the assertion can be proved we need to describe the $\sigma$-prime minimal prime ideals of each of $\mathbf{F}, \mathbf{B}$, and $\mathbf{P}$. Since $[0,1]$ is compact those of $\mathbf{F}$ are all fixed, i.e. of the form $j_{x} \cap F$ where $j_{x}=\{a \in P: x \notin a\}$, for $x \in[0,1]$. Also the non-measurability of the cardinal of $[0,1]$ implies that the $\sigma$-prime minimal prime ideals of $\mathbf{P}$ are all of the form $j_{x}$ for $x \in[0,1]$. Now all three of $\mathbf{F}, \mathbf{B}$ and $\mathbf{P}$ are perfectly normal and so Lemma 2.6 implies that every $\sigma$-prime minimal prime ideal of $\mathbf{B}$ is of the form $j_{\alpha} \cap B$ for some $x \in[0,1]$. This last result is also a consequence of $8.4[6]$.

Turning now to proving that $\mathbf{B}$ is a $z$-sublattice of $\mathbf{P}$ we note that $5.4(\alpha)$ is 
obviously true, $(\beta)$ has already been remarked upon, and so only $(\gamma)$ remains. But each singleton $\{x\}$ belongs to $B$ and so $(\gamma)$ is easily seen to be equivalent to

$$
[0,1] \backslash b=\bigcap_{x \in b}[0,1] \backslash\{x\}, b \subseteq[0,1]
$$

where the sets in the right-hand intersection are all in $B$. Thus $B$ is a proper $z$-sublattice of $\mathbf{P}$.

Our next result shows that a zero-set lattice $\mathbf{Z}(X)$ can never be a proper $z$-sublattice of a $z$-lattice, and this is the point where we can see why Gordon's results [6] differ in some respect from the usual topological ones. Simply put, his zero-set structures are more general than those which can arise in the topological context, and so a result such as: a product of pseudo-compact zero-set spaces is pseudo-compact, can be valid in the former while failing in the latter. Put another way, $z$-lattices such as the $\mathbf{B}$ of 5.7 can never arise as $\mathbf{Z}(X)$ for a topological space $X$. We note that if this could happen, results of Mandelker [10] imply that $X$ would be at least a $P$-space!

THEOREM 5.8. Suppose $X$ and $Y$ are realcompact spaces and that $Z(Y)$ is isomorphic to a $z$-sublattice $\mathbf{L}$ of $\mathbf{Z}(X)$. Then $\mathbf{Z}(Y)$ is isomorphic to $\mathbf{Z}(X)$.

Proof. We prove that $X$ and $Y$ are homeomorphic under the stated assumptions. It is easy to see that the space of $\sigma$-prime minimal prime ideals of $\mathbf{Z}(X)$ topologised as in 5.6 is canonically homeomorphic to $X$; we denote it $X^{*}$ with points $j_{x}=\{a \in Z(X): x \notin a\}$. Similarly for $Z(Y)$. Thus we have the following diagram, where $L^{\prime}=\left\{X_{a}^{Z(X)}: a \in L\right\}$ and $Z^{\prime}=\left\{X_{b}^{Z(X)}: b \in Z(X)\right\}$ :

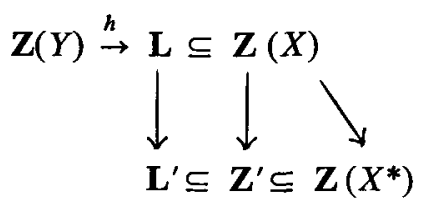

where all the maps which are not inclusions are isomorphisms; the vertical maps are as in the construction 5.6 and the diagonal map is defined using the homeomorphism $x \rightarrow j_{x}$.

Now (5.4) $(\beta)$ states that the map

$$
x^{*}=j_{x} \rightarrow j_{\checkmark} \cap L
$$

is a bijection; by construction it is continuous from $X^{*}$ onto $X^{\mathrm{L}}$. We show that it is a closed map. A typical closed subset $F$ of $X^{*}$ is of the form

$$
F=\bigcap_{b=B} X_{b}^{*}
$$

where $B \subseteq \mathbf{Z}(X)$. Condition (5.4) $(\gamma)$ states in this context that for each $b \in \mathbf{Z}(X)$, $X_{b}^{*}=\bigcap\left\{X_{a}^{*}: a \in L, a \geqq b\right\}$ whence 


$$
F=\bigcap_{a \in A} X_{a}^{*}
$$

where $A \subseteq L$. But now we may apply the bijection above, and we find that $F \rightarrow \bigcap_{a \in A} X_{a}^{\mathrm{L}}$, a closed subset of $X^{\mathrm{L}}$. Thus $X^{*}$ is homeomorphic to $X^{\mathrm{L}}$ and so we deduce that $X$ is homeomorphic to $Y$. Finally we complete the proof by noting that the lattice of zero-sets of a topological space is a topological invariant.

We can now finish off with the characterisation theorem.

THEOREM 5.9. The following are equivalent for a lattice $\mathbf{L}$.

1) $\mathbf{L}$ is isomorphic to $\mathbf{Z}(X)$ for a topological space $X$.

2) $(\alpha) \mathbf{L}$ is $(2, \sigma)$-complete;

$(\beta) \mathbf{L}$ has enough $\sigma$-prime minimal prime ideals;

$(\gamma) \mathrm{L}$ is perfectly normal;

$(\delta) \mathbf{L}$ is isomorphic to every $z$-extension $\mathbf{L}^{\prime}$ of $\mathbf{L}$.

Proof. 1) implies 2). Properties $(\alpha),(\beta)$ and $(\gamma)$ have already been observed Suppose that $\mathbf{Z}(X)$ is a $z$-sublattice of a $z$-lattice $\mathbf{L}^{\prime}$. Noting that we may suppose $X$ is realcompact by 5.2 , we have the following diagram:

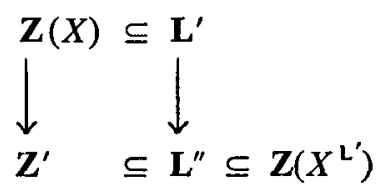

where the vertical maps are isomorphisms as in the construction of 5.6, and the horizontal maps are inclusions. By the transitivity of the property of being a $z$-sublattice, $\mathbf{Z}^{\prime}$ is a $z$-sublattice of $\mathbf{Z}\left(X^{\mathrm{L}^{\prime}}\right)$ isomorphic to $\mathbf{Z}(X)$, and so $\mathbf{Z}(X) \cong \mathbf{L}^{\prime}$ follows from 5.8 .

2) implies 1). We have already proved that if $\mathbf{L}$ satisfies 2$)(\alpha),(\beta),(\gamma), \mathbf{L}$ is isomorphic to a $z$-sublattice $\mathbf{L}^{\prime}$ of $\mathbf{Z}(X)$ for a completely regular space $X$, and so by $(\delta)$ we may conclude that $\mathbf{L} \cong \mathbf{Z}(X)$.

\section{References}

[1] G. Birkhoff, Lattice Theory Colloq. Publ. No. XXV, 3rd Ed., (Amer. Math. Soc. Providence, Rhode Island, 1967).

[2] N. Bourbaki, Elements of mathematics, General topology, Part 1 (Hermann, Paris: AddisonWesley, Reading Massachussetts, Palo Alto, London, Don Mills Ontario; 1966).

[3] E. Cech, Topological Spaces (Interscience Publishers, John Wiley and Sons, London, New York; Sydney, 1966).

[4] W. H. Cornish, 'Normal lattices', J. Austral Math. Soc. 14 (1972), 200-215.

[5] L. Gillman and M. Jerison, Rings of continuous functions (D. Van Nostrand Princeton, New Jersey, Toronto, London, New York, 1960). 
[6] H. Gordon, 'Rings of functions determined by zero sets', Pacific. J. Math. 36 (1971), 133-157.

[7] J. Kesttan, 'Eine Charakterisierung der vollständig regulären Raume', Math. Nachr. 17 (1958/ 1959), 27-46.

[8] J. Kist, 'Minimal prime ideals in commutative semigroups', Proc. London Math. Soc. (3) 13 (1963), 31-50.

[9] E. R. Lorch, 'Compactification, Baire functions, and Daniell integration', Acta. Sci. Math. Szeged, 24 (1963), 204-218.

[10] M. Mandelker, 'Relative annihilators in lattices', Duke Math. J. 37 (1970), 377-386.

[11] T. P. Speed, 'On rings of sets', J. Austral. Math. Soc. 8 (1968), 723-730.

Department of Probability of Statistics

University

Sheffield, S3 7RH, England 